\title{
What a maker's knowledge could be
}

\author{
Luciano Floridi ${ }^{1}$
}

Received: 4 March 2016 / Accepted: 26 September 2016 / Published online: 14 October 2016 (C) The Author(s) 2016. This article is published with open access at Springerlink.com

\begin{abstract}
Three classic distinctions specify that truths can be necessary versus contingent,analytic versus synthetic, and a priori versus a posteriori. The philosopher reading this article knows very well both how useful and ordinary such distinctions are in our conceptual work and that they have been subject to many and detailed debates, especially the last two. In the following pages, I do not wish to discuss how far they may be tenable. I shall assume that, if they are reasonable and non problematic in some ordinary cases, then they can be used in order to understand what kind of knowledge the maker's knowledge is. By this I mean the sort of knowledge that Alice enjoys when she holds the information (true content) that Bob's coffee is sweetened because she just put two spoons of sugar in it herself. The maker's knowledge tradition is quite important but it is not mainstream in modern and analytic epistemology and lacks grounding in terms of exactly what sort of knowledge one is talking about. My suggestion is that this grounding can be provided by a minimalist approach, based on an information-theoretical analysis. In the article, I argue that (a) we need to decouple a fourth distinction, namely informative versus uninformative, from the previous three and, in particular, from its implicit association with analytic versus synthetic and $a$ priori versus a posteriori; (b) such a decoupling facilitates, and is facilitated by, moving from a monoagent to a multiagent approach: the distinctions qualify a proposition, a message, or some information not just in themselves but relationally, with respect to an informational agent; (c) the decoupling and the multiagent approach enable a re-mapping of currently available positions in epistemology (Classic, Innatist, Kant's and Kripke's) on these four dichotomies; (d) within such a re-mapping, two positions, capturing the nature of a witness' knowledge and of a maker's knowledge,
\end{abstract}

\footnotetext{
$凶 \quad$ Luciano Floridi

luciano.floridi@oii.ox.ac.uk

1 Oxford Internet Institute, University of Oxford, 1 St Giles, Oxford OX1 3JS, UK
} 
can best be described as contingent, synthetic, a posteriori, and uninformative and as contingent, synthetic, weakly a priori (ab anteriori), and uninformative respectively. In the conclusion, I indicate why the analysis of the maker's knowledge has important consequences in all those cases in which the poietic (constructive) intervention on a system determines the truth of the model of that system.

Keywords Ab anteriori - Analytic - A posteriori - A priori - Epistemology · Information theory · Maker's knowledge · Philosophy of information - Synthetic . Witness' knowledge

\section{Maker's knowledge: the question}

Let us assume that Alice knows that $p$. And let us assume that, at least in some cases, her knowledge that $p$ can be correctly analysed as follows:

(i) she holds the information that $p ;^{1}$

(ii) $p$ is true $;^{2}$ and

(iii) she can successfully provide the right account (justification, explanation, warrant, ...) for $p$.

Clause (i) replaces the doxastic clause in the traditional Gettier-like analysis. Clause (ii) is the assumption on which all philosophers agree since Plato: truth is a necessary condition for knowledge. ${ }^{3}$ Clause (iii) expands, in a non-committal way, the kind of justification that Alice may provide. I have defended the tenability of (i)-(iii) elsewhere $^{4}$ and, in this article, I hope the reader may concede these assumptions as our starting point, at least conditionally. For the question I am keen on investigating is not whether (i)-(iii) are always correct-sometimes they are not, as Gettier-type problems show - but, rather, if they are (sometimes) correct, when they are, what happens, from an information-theoretic perspective, if Alice is also responsible for the world to be in the state modelled (captured, described, fitted, represented, ...) by $p$ ? In other words, what is the informational analysis of the so-called maker's knowledge, ${ }^{5}$ if we start from (i)-(iii)? And does the maker's knowledge that $p$ informationally differ both

1 In the rest of the article I omit "that" and write "information $p$ " not "information that $p$ " in order to indicate that $p$ is the information communicated by a message. The reader unhappy with this solution is welcome to disregard it and add a "that".

2 For supporters of the veridicality thesis ( $p$ qualifies as semantic information only if $p$ is true), like Grice, Dretske or myself, the qualification that the information that $p$ is true is redundant [see also Adams (2003)]. I provide reasons in its favour in Floridi (2007) and Floridi (2011a). Opponents of the veridicality thesis, like Colburn (2000a,b), Dodig-Crnkovic (2005), Ferguson (2015), Fetzer (2004), Fresco and Michael (2016), and Scarantino and Piccinini (2010) may find it necessary. In this article, the veridicality thesis plays no role, since the starting assumption is that Alice knows $p$, and hence any analysis of $p$, whether informational or not, must presuppose the truth of $p$.

3 So throughout this article, agents deal with information that is actually true, not with information that they think is true but may be false (Gerbrandy and Groeneveld 1997).

4 See Floridi (2011a). For a theory of truth that fits well with the veridicality thesis, see Floridi (2010), for a theory of accounting for semantic information, based on network theory, that fits both, see Floridi (2012).

5 Maker's knowledge has been understood both negatively, as a way of establishing the limits to human knowledge (one can only know what one makes) and positively, as a way of supporting human knowledge 
from the observer's (also known as witness') knowledge that $p$-i.e., Bob observing Alice interacting with the world (more on this soon) - and from the knowledge that $p$ enjoyed by a receiver of the information $p$ who does not observe that $p$, i.e. Carol, who receives a message from Bob about Alice interacting with the world?

In the previous twofold question, six elements are at play:

1. a system $S$ that changes. The easiest solution is to use a $S$ with some simple, clear, and fully accessible and controllable transition states (although it could be just any empirical state of the world, continuous or discrete). Note that the chosen system is not (always) deterministic. ${ }^{6}$ Its behaviour must be contingent in order for the agent really to be the maker of a new state: there must be some causal connection related to the choices that the maker can exercise $;^{7}$

2. an agent who changes $S$. This is Alice the maker;

3. a change ${ }^{8}$ in $S$, from $S_{1}$ to $S_{2}$, brought about by Alice. This is $s$;

4. a message that conveys $p$, where $p$, the informational content of the message, is that $s$ has occurred. This is $m$;

5. an agent who does not bring about but observes $s$. This is Bob the observer (or witness);

6. an agent who does not bring about nor observes $s$, but receives the information $p$. This is Carol the receiver, to whom Bob communicates $p$ through $m$.

These six elements look like a game and indeed an example from chess provides all we need to build a simple example:

1. $S$ : chess game

2. Alice: the white player

3. $s$ : white King's pawn moved two steps by Alice

4. $m$ : the move $s$ communicated (e.g. by voice) in English notation by Bob to Carol

5. $p$ : the true information e2-e4

6. Bob: the black player, who observes Alice's move $s$ and sends $m$ to Carol

7. Carol: the receiver of $m$.

Figure 1 shows the move on the chessboard made by Alice.

Alice has made the move, Bob has seen her making the move and communicates the move to Carol, who is in another room, by sending her $m$, the message that conveys the information $p$. In the end, all three agents hold the information $p$. Recall now that we started by assuming an analysis of knowledge comprising an information component,

\footnotetext{
Footnote 5 continued

(one effectively knows only what one creates). The history of the debate on the nature of maker's knowledge is long [see Hintikka (1974), Gaukroger (1986), Pérez-Ramos (1988), Kennington (1989), Souza Filho (2002), and Zittel (2008)] and it includes Plato, Philo of Alexandria, Bacon, Hobbes, Vico, and Kant, just to list some major philosophers. I have dealt with it in Floridi (2011b).

6 I am grateful to one of the anonymous reviewers for this important specification.

7 I am grateful to one of the anonymous reviewers for the following specification, which I quote verbatim: "It should be noted that 'states' in both digital and analogue system are artificially imposed, and so are, arguably, already part of maker's knowledge. Systems that are normally deemed digital (on/off light switches, Boolean gates, etc.) are discrete at one level of abstraction, yet analogue at another.”. I completely agree, as argued in Floridi (2011a).

8 In the literature on dynamic epistemic logic this is sometime qualified as "ontic" or "factual" change.
} 


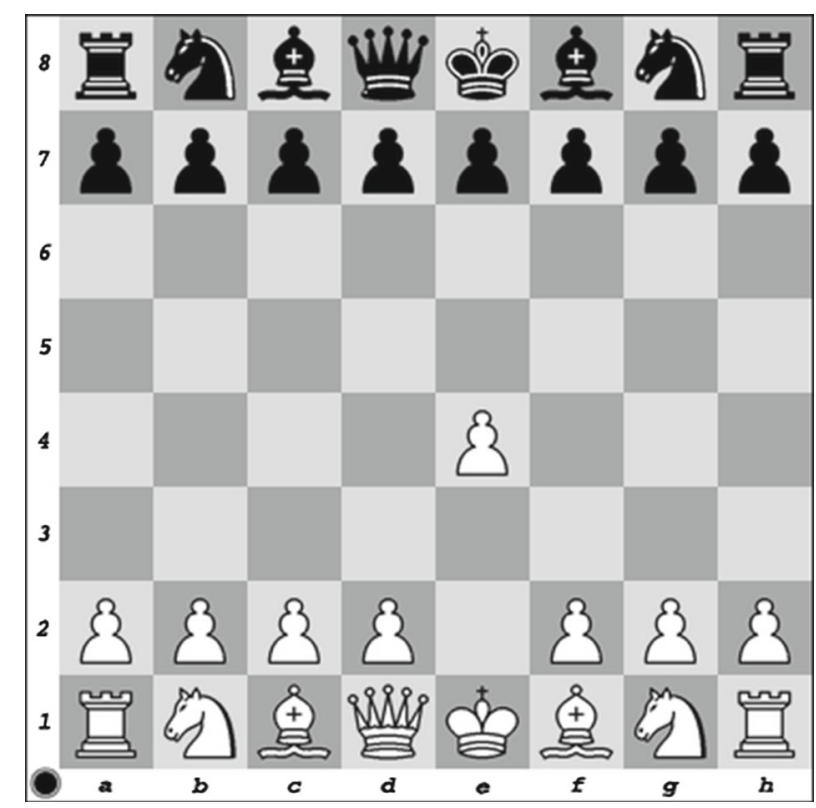

Fig. 1 Alice has moved the King's pawn two steps (e2-e4)

its truth, and a correct account component. If Alice's knowledge is different from Bob's and Carol's — since they all presuppose the truth clause (ii) — then only three possibilities present themselves: because of the information component (i), because of account component (iii) or because of both, that is

(a) because Alice's information $p$ differs;

(b) or because Alice's account for $p$ differs;

(c) or because both (a) and (b).

I shall argue in section three that (b) is the correct answer: it is the logical nature of the maker's account that differs, not the maker's information.

\section{Maker's knowledge: same information}

Let me first exclude the possibility that Alice's knowledge may differ from Bob's and Carol's knowledge because it comprises a different kind of true information. It does not. Suppose the move e2-e4 has been made. And suppose we do not know who is who. We ask all three agents to join us in a different room and broadcast $m$ to all of them. In this dynamic setting, ${ }^{9}$ we know that Carol is the only agent whose epistemic status about $s$ can be upgraded. ${ }^{10}$ This because the other two agents, independently of

\footnotetext{
9 van Ditmarsch et al. (2007) is an outstanding presentation of dynamic epistemic logic that has influenced the ideas presented in this article.

10 To be precise, since this is now a case of common knowledge, Alice's and Bob's epistemic states are also updated with respect to their information about who is informed about who is informed about $p$ (that
} 
who is Alice and who is Bob, will find $m$ redundant, as they already hold $p$, regardless of who made the move and who saw the move being made. Yet now, if Alice and Bob have a different knowledge that $p$ from Carol's, this cannot be because they have a different information $p$, so (a) cannot be the case and therefore (c) is also excluded. Agents may hold some information differently—e.g. tentatively, doubtfully, or with complete certainty - but this is how they hold it, not what they hold. If they hold $p$, it is the same $p$ that occurs. So if there is a difference between Alice's, Bob's and Carol's knowledge that $p$, it cannot be in what they hold, the information $p$. Either there is no difference, or something else must be playing a role. But we said that their epistemic states could differ only in terms of what information they hold or in terms of how they account for it. So, if there is a difference, this must be in the account that Alice, Bob, and Carol can provide of $p$. But is there a difference?

Consider Carol first. Recall that we broadcasted $m$. At that point, all Carol has is the true information $p$, nothing else. In short, Carol does not know that $p$, she is merely informed (holds the true information) that $p$, because, in our example, she has no account to provide (no justification, warrant etc.) over and above the simple fact of having received $m$ from Bob (we are excluding by design the possibility that she may appeal to Bob's reliability, for example). In more standard terminology, she believes that $p, p$ is true, but she has no justification for $p$. So the difference between Alice and Bob on the one hand, and Carol on the other is indeed one of account, because Alice and Bob can provide one and Carol cannot.

Consider Alice and Bob next. They both can provide an account for $p$. A fundamental difference between their epistemic states - and one that, if necessary, further differentiates Alice's knowledge from Carol's - is that, if Bob holds the true information $p$, then the perceptual conditions that make Bob informed that $p$-and that can provide him with the resources for an account for $p$, e.g. a reliable vision of $s$-are different from the making (henceforth poietic) conditions that make $p$ true. Bob see e2-e4 because it is true that "e2-e4", but his seeing it does not make it true. Perceiving something to be the case is different from making that something to be the case. If Bob had hallucinated "f2-f4" this would not have made any difference to the chessboard. This is an anti-idealist conclusion. Imagine next that Bob walks into the other room and communicates to Carol that "e2-e4". Thanks to Bob, Carol acquires the information $p$. Bob's information is the sender's information: he can send $m$ conveying $p$ because he already holds the information $p$, this is why our previous broadcasting was redundant for him. The further point is now that $p$ is true but not because Bob sends $m$ : sending a message $m$ conveying $p$ does not make $p$ true. If Bob had made a mistake and sent " $\mathrm{f} 2-\mathrm{f} 4$ " this too would not have made any difference to the chessboard. ${ }^{11}$

Let us return to Alice. It is clear that the difference between her knowledge and Bob's is that, in her case, if she is informed about $s$, then the experiential conditions that make Alice informed that $p$ are the same poietic conditions that make $p$ true. This is a constructionist conclusion. In the maker's knowledge case, poiesis (making $s$

\section{Footnote 10 continued}

is, everyone in the group is informed that $p$, everyone is informed that everyone is informed that $p$, and so on). However, this metaepistemic aspect is not relevant in this context and I shall disregard it.

11 As one of the anonymous reviewers correctly remarked, it would have made a difference to what $p$ is. 
happen) and alethisation (making $p$ about $s$ true) are two sides of the same coin. Alice is not merely the sender of the information that $p$, she is the source of the referent of $p .^{12}$ But then, does her account of $p$ differ from Bob's?

\section{Maker's knowledge: different account}

To understand how Alice's account (and hence her ensuing knowledge) that $p$ may differ from Bob's it is useful to rely on the three classic distinctions that can be used to qualify a truth:

(a) necessary versus contingent

(b) analytic versus synthetic

(c) a priori versus a posteriori

Such a reliance is not meant to be uncritical. The reader will know too well that the tenability of each couple has been challenged, especially (b) ${ }^{13}$ and (c). ${ }^{14}$ So what I am asking from the reader is only a conditional acceptance. If (or perhaps more optimistically insofar as) such distinctions can be reliably and usefully adopted in some cases, then I hope the reader will be willing to concede that they apply to Alice's, Bob's and Carol's knowledge that the King's pawn has moved two steps. So I do not wish to argue, for example, that we should disregard Quine's or Williamson's criticisms and accept (b) or (c) as perfectly fine. What I intend to show is that, if we are ready to use such distinctions as sufficiently unproblematic in some cases, then "e2-e4" is one of them, and yet more work needs to be done, for a further distinction needs to be introduced in order to explain how Alice, who enjoys a maker's knowledge that $p$, can account for $p$ differently from Bob and Carol. ${ }^{15}$ Let us see why.

It seems clear that Alice's knowledge about $s$ (or that $p$ ) is synthetic, not analytic, and contingent, not necessary (Floridi 2011a). This is due to the fact that the true information in question, "e2-e4", is synthetic and contingent, and that we assume that such properties are inherited by the knowledge of such truth. As they write in logic textbooks, the rest of the argument can be left as an exercise. But is Alice's knowledge a priori or a posteriori? This is tricky. The distinction has undergone many

\footnotetext{
12 As Patrick Allo has rightly remarked commenting on a previous version of this article, perhaps one could use something like Kripke's causal theory of reference to expand this dichotomy into a semantic direction, interpreting Alice's poiesis in a way akin to an "initial baptism", except she inaugurates some state of affairs rather than a name.

13 For a careful review of the debate and a defence of the tenability of the distinction against Quine's arguments see Russell (2008). On Quine's arguments based on the indeterminacy thesis I follow Pagin (2008). A very useful reconstruction of the history of the distinction in Kant and its role in his philosophy is provided by Anderson (2015).

14 For two excellent analyses to which I am indebted see Casullo (2013) and Jenkins and Kasaki (2015), see also Casullo and Thurow (2013), and Casullo (forthcoming).

15 An important topic that I hope to explore thoroughly in the future is the connection between maker's knowledge and "instructional information" (see Floridi (2011a)), "control information" [see Corning (2007)] and A. Sloman (http://www.cs.bham.ac.uk/research/projects/cogaff/misc/whats-information.html) and "information how" [see Fresco (2016)]. As one of the anonymous reviewers remarked: "In this type of information, both affordances and success criteria play a key role, and the agent exercising instructional information enjoys a special epistemic status similar to maker's knowledge".
} 
interpretations since its Kantian formulation. Here, I am happy to adopt a fairly orthodox understanding, well represented in standard introductions to philosophical logic. Many would do as well. The following comes from David Papineau's excellent book Philosophical Devices, which formulates it in terms of kinds of knowledge, exactly what we need in this context:

This distinction [between a priori and a posteriori truths] is to do with kinds of knowledge [my italics] [...]. A true statement is a priori if it can be known prior to experience of the facts. In principle, you can figure out an a priori truth just by sitting in an armchair with your eyes shut and thinking hard. The most obvious examples of a priori truths are analytic truths. Anybody who understands the statement triangles have three sides won't need to examine any physical triangle to know that this statement is true. [...] A true statement is a posteriori if it can only be known as a result of relevant experience. Blackbirds eat worms is an example of an a posteriori truth. There is no way of finding out that this statement is true without making observations. (Papineau 2012), p. 46.

Consider Alice's knowledge that "e2-e4" in the light of the previous distinction.

On the one hand, it does not seem a posteriori, at least not in the same sense in which Bob's is. This because, if Alice has a "way of finding out that this statement is true without making observations", this is by being successful in moving the pawn. If this is unclear, or seems an extraordinary case, consider another simple scenario. Alice is alone in the kitchen. She makes some coffee for Bob and puts two spoons of sugar in it. Bob comes into the kitchen and wishes to know whether the coffee has been sweetened. He can only know that a posteriori, "as a result of relevant experience", as Papineau puts it: by tasting the coffee or by asking Alice (the latter would be a case represented by Carol, in our chess example, i.e. knowledge through communication rather than empirical experience). Yet Alice does not need to taste the coffee in order to know the truth of the statement "the coffee has been sweetened". Barring any sceptical scenario, Gettierization, or empirical mistake, ${ }^{16}$ if Alice knows that "e2-e4" then her account for the fact that "e2-e4" or that "the coffee has been sweetened" is acquired through experience as interaction, ${ }^{17}$ that is, by changing the world, and not by following experience as perception; whereas Bob's is acquired by experience as perception, that is, by observing the world without changing it: and Carol's is acquired by proxy experience as communication, that is, by relying on Bob's perception. Alice changes the world and insofar as she succeeds (and we assume that she does, because we assumed that she knows) this is how she knows the world is.

On the other hand, Alice's knowledge may seem to be a priori, insofar as this is simply a negation of a posteriori. However, this too is not entirely satisfactory. Admittedly, her knowledge that the King's pawn has moved two steps-if it is knowledge (and we assume that it is) - is based on the fact that she meant to move it that way

\footnotetext{
16 These three issues are out of the question here by hypothesis. Recall that we are assuming that Alice does know that $p$, we are trying to understand in which way, if any, her knowledge differs from Bob's, who is also assumed to know that $p$.

17 As a consequence, the position defended in this article is compatible with a reliabilist epistemology of justification that does not reject the a priori versus a posteriori distinction, see Grundmann (2015).
} 
and succeeded in doing so-again, her success is not in question, it is assumed by hypothesis - and this is the source of the kind of account that she can provide. For example, to the question how does she know that the pawn has moved two steps, she could simply answer that she moved it herself, and that she did so because of a particular strategy. ${ }^{18}$ Likewise, if she knows that the coffee has been sweetened she does so, in terms of having an account for her true information that is somehow (more on this crucial qualification presently) "prior to experience of the facts" (to quote Papineau again), the sort of experience that Bob requires to know that the coffee has been sweetened; or in advance, so to speak (again, more on this presently), because that was her plan, and she successfully carried it out. In a more standard vocabulary, $p$ is true, Alice believes that $p$, and she is justified in believing that $p$ because (or, equivalently: her justification is based on the fact that) she is the one who brought about the truth of $p$ (made $p$ true) in the first place. However, it also seems clear that her knowledge of "e2-e4" is different from Carol's a priori knowledge that "the pawn that was moved was either black or white", which requires no interactive or observational experience to be acquired. To use Papineau's analysis once more, in principle, Carol having been told that a pawn has been moved, she can figure out that the moved pawn is either black or white by sitting in an armchair with her eyes shut and thinking hard, by merely knowing the rules of chess. Alice's information about $s$ is the source's (not merely the sender's) information: it is synthetic, contingent, and not a posteriori. But it is a priori only in a weaker sense than the one usually described in textbooks. In order to provide an account of $s$, Alice does not need to consult experience at all. Actually, her account for making $s$ the case comes logically before $p$ being true. She might want to refer, for example, to a particular opening strategy that she intends to pursue by making that move, and predict that the world will be such as to satisfy the truth of "e2-e4" by executing her action plan.

One may object that making $s$ happen and therefore being informed that $p$ because of such a making is intertwined with the perception that one is making $s$ happen. This is correct. Alice also enjoys the same knowledge that Bob has, insofar as she is also an observer of her own move. ${ }^{19}$ This means that Alice may no longer see clearly whether she knows that $p$ because she made $s$ happen (because of her interaction with the world) or because she perceived herself making $s$ happen (because of her perception of the

\footnotetext{
18 I agree with one of the anonymous referees who suggested that "Arguably, the different justification that Alice might give has to do with her disposition, plan, desire, belief, etc. that making p occur will succeed. [...] Alice's and Bob's perception of $p$ may be attributed [in prediction error (PE) minimisation theory] to $\mathrm{PE}=0$ for their predictions that $p$ is the case. The privileged cognitive resource Alice enjoys, but not Bob, is her intent to act on the King's pawn to make it such that $p$ will be true. Her prediction that $p$ is likely to be more accurate/reliable than Bob's-absent overriding factors in the environment that interfere with Alice's making $p$ true."

19 Note that Alice need not be informed about the fact that she is informed about $s$. The fact that the analysis developed in this article does not require the acceptance of the KK (or in this context II) thesis (aka $\mathrm{S} 4$, K3, or the reflective thesis about positive introspection), namely $\square \varphi \rightarrow \square \square \varphi$, is consistent with the modal logic KTB (aka B), which I have argued, in Floridi (2006), may be suitable as a logic for "S holds the information $p$ ". The consistency of the previous analysis with KTB does not preclude the possibility of $\square \square \varphi$. On the contrary, the message $m$ can work as a trigger for both Alice and Bob to make possible, and indeed facilitate, $\square \square \varphi$. I have defended this possibility in terms of a double channel explanation of KK (or II) in Floridi (2006).
} 
world and her interaction with it). In our example, the solution is to decouple making $s$ happen and perceiving oneself making $s$ happen. This may be problematic, but it is doable. For example, Alice may control Bob's chessboard remotely (correspondence chess). If she knows that Bob's chessboard is in state $s$, then this is so even if she cannot perceive herself making a difference to it.

Let me summarise what conclusions we have reached so far.

First, Alice (the maker) enjoys the same synthetic and contingent information about $s$ as Bob and Carol do. The difference among the three agents is not one in terms of different kinds of information (or different kinds of beliefs, or different kinds of propositional contents, to use a more textbook vocabulary). Perhaps this explains epistemology's disinterest in the maker's knowledge tradition. In terms of kinds of information (the what is hold not the how or why), there is no distinction, so there is no interesting theory to develop about this.

Second, it seems that Alice's knowledge is also not a posteriori, at least not in the same sense in which Bob's and Carol's is, because she also enjoys a source's weak (a full qualification still pending) a priori account for the information about $s$ that she brings about, which both Bob the observer and Carol the receiver of Bob's message lack by definition. Perhaps this explains logic's disinterest in the maker's knowledge tradition:

Since DEL [dynamic epistemic logic] is mostly about information change due to communication, the model transformations usually do not involve factual change. The bare physical facts of the world remain unchanged, but the agents' information about the world changes. In terms of Kripke models that means that the accessibility relations of the agents have to change (and consequently the set of states of the model might change as well). Modal operators in dynamic epistemic languages denote these model transformations". (van Ditmarsch 2015, pp. 1-2).

The case in which Alice brings about a change in the system is similar to the case in which Alice assigns a particular value to a state of the world. Factual or ontic changes can be modelled by epistemic dynamic logics that include assignment operators in the language for epistemic actions. ${ }^{20}$ However, such logics investigate the informational implications of such changes for the agents involved, not what it means for the agent who brings about the change to be able to account for it. In particular, public assignments are such that the whole group of agents is aware of the factual change-compare this to the case in which Alice moves the pawn and both her and Bob then hold the new information that the pawn has been moved-whereas atomic assignments are such that only facts are changed, independently of whether (or which) agents are informed about such changes-compare this to the case in which Alice sweetens the coffee but Bob does not know about it.

The outcome of the previous points is that a maker's knowledge looks like a hybrid: synthetic and contingent information + a weak a priori account. Is there room for this poietic knowledge in the overall map of distinctions logically available? The answer

20 The classic reference is van Ditmarsch et al. (2005), see also van Benthem et al. (2006). 


\begin{tabular}{|c|c|c|c|c|c|c|c|c|c|c|c|c|c|c|c|c|}
\hline & $\mathbf{a}$ & b & $\mathbf{c}$ & d & $\mathbf{e}$ & f & $\mathbf{g}$ & $\mathbf{h}$ & $\mathbf{i}^{\mathbf{a}}$ & 1 & $\mathbf{m}$ & $\mathbf{n}$ & $\mathbf{0}$ & $\mathbf{p}$ & & $\mathbf{r}$ \\
\hline & $\mathrm{C}$ & & & & & $\mathrm{Kr}$ & & & & $\mathrm{Ka}$ & $\mathrm{A}$ & In & & & & $\mathrm{C}$ \\
\hline Analytic & 1 & 1 & 1 & 1 & 1 & 1 & 1 & 1 & 0 & 0 & 0 & 0 & 0 & 0 & 0 & 0 \\
\hline A priori & 1 & 1 & 1 & 1 & 0 & 0 & 0 & 0 & 1 & 1 & 1 & 1 & 0 & 0 & 0 & 0 \\
\hline Necessary & 1 & 1 & 0 & 0 & 1 & 1 & 0 & 0 & 1 & 1 & 0 & 0 & 1 & 1 & 0 & 0 \\
\hline Uninformative & 1 & 0 & 1 & 0 & 1 & 0 & 1 & 0 & 1 & 0 & 1 & 0 & 1 & 0 & 1 & 0 \\
\hline Synthetic & 0 & 0 & 0 & 0 & 0 & 0 & 0 & 0 & 1 & 1 & 1 & 1 & 1 & 1 & 1 & 1 \\
\hline A posteriori & 0 & 0 & 0 & 0 & 1 & 1 & 1 & 1 & 0 & 0 & 0 & 0 & 1 & 1 & 1 & 1 \\
\hline Contingent & 0 & 0 & 1 & 1 & 0 & 0 & 1 & 1 & 0 & 0 & 1 & 1 & 0 & 0 & 1 & 1 \\
\hline Informative & 0 & 1 & 0 & 1 & 0 & 1 & 0 & 1 & 0 & 1 & 0 & 1 & 0 & 1 & 0 & 1 \\
\hline
\end{tabular}

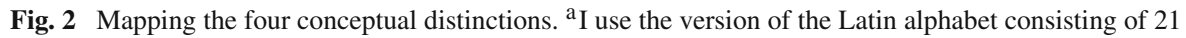
letters in order to avoid potential confusions regarding the occurrence of $\mathrm{k}$

is yes but, in order to clarify how, I need to add a further conceptual distinction and finally explain what I mean above by "somehow", "weak", and "in a sense".

\section{Maker's knowledge: ab anteriori}

Since at least Kant, philosophers have been implicitly using information, and its cognate terms, as the neutral vocabulary to discuss the nature of the three classic couples introduced above. Information has played the role of the least common denominator through which all other epistemic currencies have been related to one another. Or, to use a different metaphor, information has played the role of the perfect butler in an Oxford college: does all the conceptual work impeccably but also invisibly. If we acknowledge information's key role and bring its conceptual contribution to light by formulating it explicitly, in terms of whether a truth is also informative ${ }^{21}$ or not, over and above whether it shares any of the other three features, the result is the mapping presented in Fig. 2. Mind that the table has a twofold goal: it clarifies the various positions one can take on the dichotomies as well as showing what happens if the a priori versus a posteriori dichotomy is extended to map the couple informative versus uninformative explicitly.

A few comments are in order. We have 16 possible combinations. ${ }^{22}$ Columns a and $\mathbf{r}$ represent the classic positions. They also represent Carol's predicament. Carol's knowledge of the moves being played in the other room is either analytic, a priori,

\footnotetext{
21 By "informative" I mean "that says something new about the way the world is".

22 An interesting question, to be left unanswered in this article, is whether some combinations are provably impossible.
} 
necessary but also uninformative, that is, it is entirely and only based on her knowledge of the rules of the game (e.g. she knows that if castling has occurred then the squares between the king and the rook involved were unoccupied); or it is synthetic, a posteriori, contingent, and informative, yet this is the case only when she receives a message from Bob. Interestingly, the classic, pre-Kantian position and Carol's (the message-receiver's) position are the same, hence the single label C. Centuries before Shannon and any mathematical theory of communication, Francis Bacon's concept of vexation of nature, for example, already fit very well with $\mathrm{C}$, and this because classic epistemology is largely based on a passive, message-receiving model of information gathering, no matter whether one is in Plato's cave or in front of Descartes' fire. Note also that column a, obtained by adding "uninformativeness" as an explicit feature of a truth, is the source of the scandal of deduction, as Hintikka labelled it: mathematical truths seem to be analytic, a priori, necessary, but also uninformative. ${ }^{23}$

Column $\mathbf{n}$ was popular among Platonists and some modern defenders of innate truths, hence the label In. Both C and In were challenged by Kant, to make room for the synthetic a priori (column l). Whether successfully is open to debate ("to say the least" a critic may add). More recently, Kripke's analysis made room for the analytic a posteriori (column $\mathbf{f}$ ). The debates on $\mathbf{I}$ and $\mathbf{f}$ are still lively. From Fig. 2 it is easy to see how Kant and Kripke hold inverted positions with respect to the synthetic-analytic and a posteriori-a priori nature of the truths in question. Two assumptions shared by Kant and Kripke, and indeed by the debate on the synthetic a priori and the analytic a posteriori, are that:

- the truths in question are informational (they say something about the way the world is, e.g. what the chess move was, or whether the coffee was sweetened) and - a single agent holds such truths.

The latter point is crucial. The assumption is that what is predicated of one holder can be predicated of any holder of such truths. Concentrating only on a single, epistemic agent is fine, insofar as the couple necessary versus contingent and analytic versus synthetic are concerned. This is so because we saw that these properties qualify the nature of the truths in question first, and then the nature of the knowledge of them derivatively. But a monoagent approach is insufficient when it comes to the other two couples, for at least two main reasons.

First, because different epistemic agents may have different ways of accessing a truth, and I mean this in principle, irrespective of individual idiosyncrasies. We saw that our three abstract and idealised epistemic agents Alice, Bob and Carol have different ways of accessing the same information "e2-e4": through experience (by making $s$ to be the case), by experience (by observing that $s$ is the case) and by proxy experience (by being communicated that $s$ is the case). The epistemology of trust, witnessing, and testimony, for example, hinges on the distinction between Alice's and Bob's knowledge that "e2-e4" on the one hand, and Carol's knowledge on the other.

And second, because whether a truth-which is informational about the world, i.e. it tells one something about how the world is-is also informative-i.e., it tells

23 The reader interested in knowing more about the scandal of deduction may wish to read D'Agostino and Floridi (2009). 
one something new about how the world is, depends on the epistemic status of the epistemic agent, and this may vary from Alice, to Bob, to Carol, again in principle, not in terms of contingent idiosyncrasies. ${ }^{24}$ We saw that broadcasting $m$ does not upgrade Alice's or Bob's epistemic status. Neither the source nor the sender of the message $m$ are informed by $m$, only the receiver can be. Likewise, being the agent that brings about $s$ that makes $p$ true means having a different access to the truth of $p$ from that enjoyed by an agent who merely observes that $p .{ }^{25}$ Thus, adopting a multiagent perspective and accepting that different agents may have different kinds of access to the same truth enables one to make sense of several other columns in Fig. 2. In particular, one can see that Bob's position is represented by q. For Bob, who saw the move, the truth "e2-e4" is synthetic, a posteriori, contingent, but also uninformative, as uninformative as "bachelors are unmarried men". His correct reaction, if we were to tell him "look, Alice moved the King's white pawn from e2 to e4", would be "I know, I saw it, you are not telling me anything new". The synthetic uninformative characterises all messages that an epistemic agent receives about states of the world that such an epistemic agent has already witnessed directly. They belong to the logic of communication (redundancy) and confirmation, but not to the logic of upgrade. A crucial clarification is in order here. ${ }^{26}$

An interesting limitation of dynamic epistemic logic is that it is typically presented as a logic of communication, but does not in fact have any explicit way of talking about the "source" of a message. Strictly speaking, the senders are outside the system, and can only be simulated through the content of the message. In our example, if Bob informs Carol that $p$, this will be modelled as a message that says that Bob knows $p$, yet this message could equally well be sent by an outsider. A corollary of this feature is that observations can be modelled in exactly the same way (a message sent by the system, rather than by an observer of the system). This is very clear in puzzles like that of the wise men or the muddy children Floridi (2005). A result of this limitation is that (when seen through the eyes of DEL), the difference between how Bob and Carol came to know that $p$ becomes purely temporal: the difference in account/access is merely a difference in who got the information first. On the one hand this does not pose a problem for the position defended in this article; it is just more evidence in favour of the fact that a pure logic of information cannot be used to distinguish between knowledge acquired by observation and knowledge acquired by testimony. On the other hand, the focus on the "uninformative" makes the (undesirable) temporal reading very plausible. So let me stress that the core difference here is between messages from the system (i.e. observation) and messages from other observers of the system (i.e. communication in the intuitive sense). This is why I wrote "already witnessed directly". To put it differently: in the commutative diagram below (Fig. 3) observation matches vertical (model-tosystem) arrows, but communication matches horizontal (model-to-model) arrows.

\footnotetext{
24 This is not a reference to utility but to upgradability: if Alice does not have the information that "Paris is the capital of France" and Bob does, then if both are told that Paris is the capital of France this is informative for Alice but not for Bob.

25 E.g. in terms of predictability of the truth, see note above on prediction error (PE) minimisation theory.

26 I am most grateful to Patrick Allo for this point and its precise formulation because, left implicit, it may mislead more than one reader.
} 


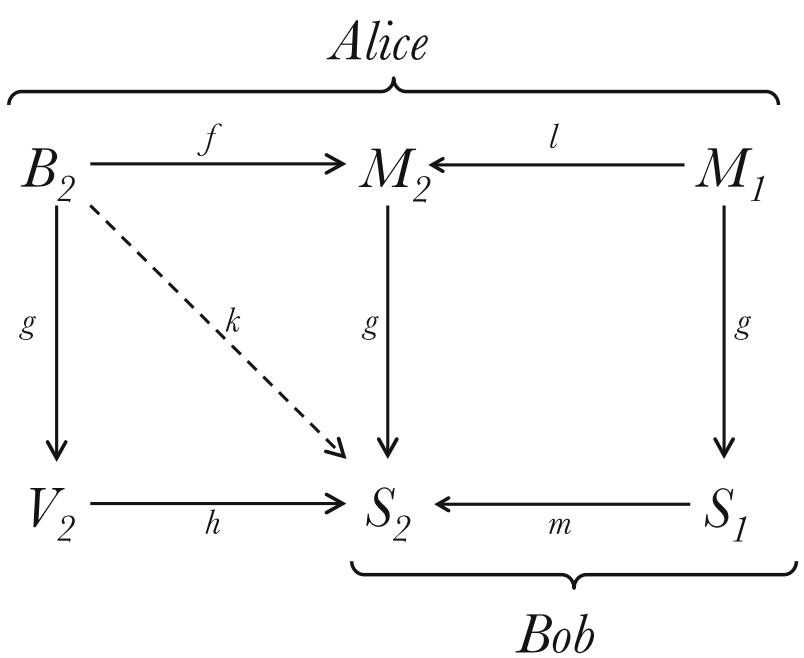

Fig. 3 Commutative diagram for the Maker's and the Witness's knowledge

Finally, we can make sense of Alice's (the maker's) knowledge, in terms of column m. By drawing a distinction between $a$ priori and a posteriori as if the two were a binary, mutually exclusive couple, we miss the fact that quite a lot happens between the two, neither before nor after but through experience. ${ }^{27}$ There is no technical term for the somewhat weak a priori knowledge that $p$ enjoyed by an agent responsible for bringing about the truth of $p$. So I shall use $a b$ anteriori to refer to such a third space. We are now ready for a definition:

The maker's knowledge is ab anteriori knowledge. A contingent, synthetic proposition $p$ about $s$ (the true information $p$ ) is an ab anteriori truth if and only if it can be known by interacting with $s$ to make $p$ true.

In principle, you can figure out an ab anteriori truth just by changing the world so that it makes the relevant statement true (it fits it). Among the most obvious examples of ab anteriori truths are performatives, understood in the way (Searle 1989) does, not Austin. Anybody who understands the statement "you are fired" uttered successfully will not need to check any facts to know how the world has changed accordingly, and hence that this statement is true. Note that, given the previous definition, any contingent, synthetic information the truth of which cannot be brought about by interacting with the system it refers to, must be a posteriori, if Kant is wrong.

The last step to be taken is to provide a clear representation of Alice's ab anteriori knowledge. To do this I shall adapt the concept of a commutative diagram ${ }^{28}$ from category theory. Consider Fig. 3.

On the right side of the diagram, Bob observes the system as it (ontically or factually) changes $(m)$ from $S_{1}$ to $S_{2}$, and he updates informationally $(l)$ his model (representa-

\footnotetext{
27 For similar lines of criticism see Jenkins (2008) and Casullo (2013).

28 The diagram is adapted from the entry "commutative diagram" in Wikipedia: https://en.wikipedia.org/ wiki/Commutative_diagram. The only difference is that the original diagram does not have the dotted arrow connecting $\mathrm{B}_{2}$ to $\mathrm{S}_{2}$. This is adapted from the first isomorphism theorem, also explained in the entry.
} 
tion) $M_{1}$, which fits $(g) S_{1}$, into his model (representation) $M_{2}$, which fits $(g) S_{2}$. Bob's access to $S$ states commutes: $m \circ g=g \circ l$. His side of the diagram can be translated into a standard Kripke model in dynamic epistemic logic.

Insofar as Alice is also an observer of the same transition, she has the same epistemic access to $m \circ g=g \circ l$ that Bob has. However, on the left side of the diagram, Alice the maker observes the system as it (ontically or factually) implements $(h)$ the virtual system $V_{2}$ (e.g. the possible move e2-e4) into the system $S_{2}$ (in our example, the actual state of the chessboard after her e2-e4 move) thanks to her interactions, and she updates informationally her blueprint (in our example, her strategy to move e2-e4) $B_{2}$, which fits $(g) V_{2}$ and fits ${ }^{29}(k) S_{2}$, into her model (representation) $M_{2}$, which fits ( $g$ ) $S_{2}$. Alice's information still commutes: $h \circ g=k=g \circ f$. The difference is that now she has a maker's access to $S_{2}$ that Bob cannot have, and can account for the information $p$ in a way that is unavailable to Bob. Finally, the reader may recall the case in which Alice was playing correspondence chess with Bob. This is represented by Alice having access only to the left side of the diagram, not the right side.

\section{Conclusion}

By way of conclusion, let me now highlight a few interesting consequences of the previous analysis.

(1) It seems clear that references to experience in terms of dependence-independence or before-after, from Kant onwards, are too-coarsely grained when it comes to understanding the "riority" (a priori, a posteriori, ab anteriori) of a truth. For in Carol's case we are talking of experience socially and in terms of communication, as trust in testimony; in Bob's case, we are talking of experience epistemically, in terms of perception; and in Alice's case, we are talking about experience pragmatically, in terms of interaction. These are different senses, which explain why we need more than the equally coarsely grained dichotomy between a priori and a posteriori. For it depends on what kind of experience plays which role, and in which logical order. In more technical terms, we need a better level of abstraction Floridi (2008) lest the ab anteriority of the maker's knowledge escapes our analysis.

(2) The definition of what counts as maker's knowledge provides us with a criterion to identify who is the maker in a group of agents, that is, it enables us to identify who plays Alice. For we can exclude first all the agents whose information state can be upgraded by broadcasting the truth in question, and then all the remaining agents whose account for the truth in question can only be based on perceptual experience. If anyone is left, that is the maker. Observations in science may seem to admit no makers, and the passive tradition in epistemology, according to which epistemic agents are mere observers of the world, finds some roots in this, but much of modern and contemporary science is actually interactive-think for example

29 I use "backward-fits" to indicate that a blueprint is a project that fits a system that does not exist but that will exist if the blueprint is implemented. A model is a project that forward-fits a system that already exists. 
of CERN's main function, which is to provide the particle accelerators and other infrastructure needed for high-energy physics research-and requires a better understanding of the maker's knowledge.

(3) We saw that neither Alice nor Bob can be informed by $m$ and this is consistent with an analysis of perception in which the mutual information-in the technical sense of the expression, see (Cover and Thomas 2006) - between the state of the system (e.g. pawn moved) and the information about that state (e.g. e2-e4) is 1. I have explored this point in Floridi (2014).

(4) Bob and Carol are both outside the system producing $s$ and hence making e2e4 true. Alice is part of the system. As the ontic or factual source of $s$, Alice holds the information $p$ as part of the system that brings about $s$. It follows that the maker's knowledge is knowledge of a system from within, not from without, consistent with the Aristotelian view that "scientia est scire per causas". This too is in stark contrast with the passive view we inherit from some modern epistemology, and especially from the Cartesian tradition, which is based on a receptive perception of the world, rather than design, intervention, interaction, and control. The problem emerged in nineteenth century German philosophy of technology, which correctly criticised Kant precisely because of his inability to see that, through technology, agents create, design, and manipulate objects in themselves, not just their phenomenal perceptions. When Alice eats an apple, she eats a noumenal something, whatever else that something may be in itself. Likewise, when she builds an Ikea table, she is building a noumenal something, whatever else that something may be in itself.

(5) When only the observer's knowledge is possible, Nature replaces Alice. Imagine the case in which the wind moves the pawn two steps. Teleological interpretations of Nature (or God) the Maker tend to look for the ultimate blueprint of the universe.

(6) Most of classic epistemology has focused on a single agent. This is fine, but too restrictive in a world that needs to understand how knowledge and information work within multiagent systems. The previous analysis shows how different even our most fundamental distinctions appear, once we introduce a multiagent perspective.

Acknowledgements I discussed several versions of this article on many occasions and I am deeply indebted for their feedback to many colleagues and members of the audiences. In particular, I wish to thank Patrick Allo, Carla Canullo, Letizia Coccia, Marcello D’Agostino, Amos Golan, Erik J. Olsson, Massimo Mugnai, Marco Santambrogio, Corrado Sinigaglia, David Watson, and Frank Zenker for their insightful comments and the opportunities they kindly provided to discuss the ideas presented in this article. The following meetings were especially useful for improving the final version: a presentation, discussed during the Fifth Philosophy of Information Workshop I organised at the University of Hertfordshire (Hatfield, 27-28 March, 2013), and then the following invited talks and keynotes given at the Philosophy of Information Workshop on "The Value of Information", American University Infometrics Institute (Washington, 26 April, 2013); at the Philosophy of Information Workshop, Lund University (Lund, 10 May, 2013); at the Conference on "Ermeneutica e Interculturalità" (Università Statale di Macerata, 9-10 September, 2013); at the "Workshop Synthetic versus Analytic", Università Statale di Ferrara (Ferrara, 10 October, 2014); and the Cognition in Action Lecture given at the Università Statale di Milano (Milan, 21 March, 2014). Patrick Allo offered some very insightful and crucial comments that "penultimated" the last version. I am grateful to David Sutcliffe for his editorial revisions and to the two anonymous reviewers, who provided many and very extensive comments. 
Open Access This article is distributed under the terms of the Creative Commons Attribution 4.0 International License (http://creativecommons.org/licenses/by/4.0/), which permits unrestricted use, distribution, and reproduction in any medium, provided you give appropriate credit to the original author(s) and the source, provide a link to the Creative Commons license, and indicate if changes were made.

\section{References}

Adams, F. R. (2003). The informational turn in philosophy. Minds and Machines, 13(4), 471-501.

Anderson, R. L. (2015). The poverty of conceptual truth: Kant's analytic/synthetic distinction and the limits of metaphysics. Oxford: Oxford University Press.

Casullo, A. (2013). Four challenges to the a priori: A posteriori distinction. Synthese, 192(9), 1-24.

Casullo, A. (forthcoming). A defense of the significance of the a priori: A posteriori distinction.

Casullo, A., \& Thurow, J. C. (2013). The a priori in philosophy. Oxford: Oxford University Press.

Colburn, T. R. (2000a). Philosophy and computer science. Armonk, NY: M.E. Sharpe.

Colburn, T. R. (2000b). Information thought, and knowledge. In Proceedings of the world multiconference on systemics, cybernetics and informatics (Vol. 10, pp. 467-471).

Corning, P. A. (2007). Control information theory: The 'missing link' in the science of cybernetics. Systems Research and Behavioral Science, 24(3), 297-311.

Cover, T. M., \& Thomas, J. A. (2006). Elements of Information Theory (2nd ed.). Hoboken, NJ: Wiley.

D’Agostino, M., \& Floridi, L. (2009). The enduring scandal of deduction. Is propositional logic really uninformative? Synthese, 167(2), 271-315.

de Souza Filho, D. M. (2002). The maker's knowledge principle and the limits of science. Proceedings of the American Catholic Philosophical Association, 76, 229-237.

Dodig-Crnkovic, G. (2005). System modeling and information semantics. In Bubenko, J. et al. (eds.) Proceedings of the fifth promote IT conference. Borlänge: Studentlitteratur.

Ferguson, T. M. (2015). Two paradoxes of semantic information. Synthese, 192(11), 3719-3730.

Fetzer, J. H. (2004). Information, misinformation, and disinformation. Minds and Machines, 14(2), 223-229.

Floridi, L. (2005). Consciousness, agents and the knowledge game. Minds and Machines, 15(3-4), 415-444.

Floridi, L. (2006). The logic of being informed. Logique et Analyse, 49(196), 433-460.

Floridi, L. (2007). In defence of the veridical nature of semantic information. European Journal of Analytic Philosophy, 3(1), 31-41.

Floridi, L. (2008). The method of levels of abstraction. Minds and Machines, 18(3), 303-329.

Floridi, L. (2010). Semantic information and the correctness theory of truth. Erkenntnis, 74(2), 147-175.

Floridi, L. (2011a). The philosophy of information. Oxford: Oxford University Press.

Floridi, L. (2011b). A defence of constructionism: Philosophy as conceptual engineering. Metaphilosophy, 42(3), 282-304.

Floridi, L. (2012). Semantic information and the network theory of account. Synthese, 184(3), 431-454.

Floridi, L. (2014). Perception and testimony as data providers. Logique et Analyse, 57(226), 151-179.

Fresco, N. (2016). Information-how. Australasian Journal of Philosophy, 94(1), 58-78.

Fresco, N., \& Michael, M. (2016). Information and veridicality: Information processing and the BarHillel/Carnap paradox. Philosophy of Science, 83(1), 131-151.

Gaukroger, S. (1986). Vico and the maker's knowledge principle. History of Philosophy Quarterly, 3(1), 29-44.

Gerbrandy, J., \& Groeneveld, W. (1997). Reasoning about information change. Journal of Logic, Language and Information, 6(2), 147-169.

Grundmann, T. (2015). How reliabilism saves the apriori/aposteriori distinction. Synthese, 192(9), 27472768.

Hintikka, J. (1974). Knowledge and the known : Historical perspectives in epistemology. Dordrecht: Reidel.

Jenkins, C. S. I. (2008). Grounding concepts: An empirical basis for arithmetic knowledge. Oxford: Oxford University Press.

Jenkins, C. S. I., \& Kasaki, M. (2015). The traditional conception of the a priori. Synthese, 192(9), 27252746.

Kennington, R. (1989). Francis Bacon's idea of science and the maker's knowledge tradition. Review of Metaphysics, 43(2), 414-417.

Pagin, P. (2008). Indeterminacy and the analytic/synthetic distinctions: A survey. Synthese, 164(1), 1-18. 
Papineau, D. (2012). Philosophical devices: Proofs, probabilities, possibilities, and sets. Oxford: Oxford University Press.

Pérez-Ramos, A. (1988). Francis Bacon's idea of science and the maker's knowledge tradition. Oxford: Clarendon Press; Oxford University Press.

Russell, G. (2008). Truth in virtue of meaning: A defence of the analytic/synthetic distinction. Oxford: Oxford University Press.

Scarantino, A., \& Piccinini, G. (2010). Information without Truth. Metaphilosophy, 41(3), 313-330.

Searle, J. R. (1989). How performatives work. Linguistics and Philosophy, 12(5), 535-558.

van Benthem, J., van Eijck, J., \& Kooi, B. (2006). Logics of communication and change. Information and Computation, 204(11), 1620-1662.

van Ditmarsch, H., van der Hoek, W., \& Kooi, B. (2007). Dynamic epistemic logic. New York: Springer.

van Ditmarsch, H., van der Hoek, W., \& Kooi, B. (2015). Dynamic epistemic logic. The Internet Encyclopedia of Philosophy. Retrieved from http://www.iep.utm.edu/de-logic/.

van Ditmarsch, H. P., van der Hoek, W., \& Kooi, B. P. (2005). Dynamic epistemic logic with assignment. In ACM - Proceedings of the fourth international joint conference on autonomous agents and multiagent systems (pp. 141-148).

Zittel, C. (2008). Philosophies of technology: Francis Bacon and his contemporaries. Leiden: Brill. 